\title{
Writing Women in Mathematics into Wikipedia
}

\author{
Marie A. Vitulli
}

\author{
Communicated by Harriet Pollatsek
}

ABSTRACT. In this article I reflect upon the problems connected with writing women in mathematics into Wikipedia. I hope to persuade the reader to start editing existing Wikipedia articles and to create new articles. I also hope to persuade existing editors to contribute new articles about women mathematicians.

The twin problems of the paucity of women subjects and scarcity of women editors on Wikipedia are well known, but no solutions are on the horizon. We can nevertheless take small steps to address these problems. In response to my concerns about these problems and in order to gain first-hand experience, I became a Wikipedia editor in 2013. In the first section, I discuss gender bias on Wikipedia and the underrepresentation of women subjects. In the second section, I discuss the scarcity of women contributors and my personal experiences of the difficulties women contributors encounter. In the third section, I discuss some recent steps taken towards solving the problems, including the Wikipedia Year of Science, the related projects WikiProject Women scientists and WikiProject Women in Red, and a recent partnership between the Association for Women in Mathematics (AWM) and the Wiki Education Foundation to write and improve pages on women mathematicians. Advice for writing a biography on Wikipedia may be found in a longer, posted version of this article [3].

Marie A. Vitulli is professor emerita at the University of Oregon. Her email address is vi tu $11 \mathrm{i} @ u$ uregon. edu. This article updates and expands upon an article in the May-June 2014 AWM Newsletter www . drivehq. com/folder/p8755087/11874396230.aspx.

For permission to reprint this article, please contact: reprint-permission@ams . org.

DOI: http://dx.doi.org/10.1090/noti1650

\section{The Paucity of Women Subjects}

There have been numerous allegations that Wikipedia suffers from systemic gender bias with respect to both content and editors and that the attempts to increase women's participation have failed. ${ }^{1}$

One disturbing manifestation of the underrepresentation of women in Wikipedia is the dearth of biographies of notable women. According to WikiProject Women in Red, only $16.36 \%$ of the biographies in English Wikipedia were about women as of August 7, 2016. This is up from just over 15\% in November 2014. Wikipedia has guidelines on academic notability (also called the professor test) that a subject must meet to merit a Wikipedia page. The first two of the nine criteria of academic notability are: the person's research has made significant impact in their scholarly discipline, broadly construed, as demonstrated by independent reliable sources; and the person has received a highly prestigious academic award or honor at a national or international level. Some of the criteria are subjective in nature, and it is not surprising that some of the American mathematicians featured on Wikipedia are more notable than others.

In 2013 the National Science Foundation awarded a grant ${ }^{2}$ to Yale University to explore potential gender differences in indicators of academic notability, networks that facilitate content creation, gate-keeping processes that challenge content associated with women and scholarship, and the unintended consequences of Wikipedia's policies.

When I first perused the page for the "Category: Women mathematicians" (formerly called "Category: Female mathematicians") several years ago, I was disheartened to see how many prominent women lacked a Wikipedia page.

${ }^{1}$ For references and further discussion, see the Wikipedia page "Gender bias on Wikipedia" en.wikipedia.org/wiki/ Gender_bias_on_Wikipedia.

${ }^{2}$ NSF Sociology, Science, Technology \& Society Program Award www.nsf.gov/awardsearch/showAward?AWD_ID=1322934. 
Comparing the category lists in August of 2015 and August of 2016, I was pleased to discover that there were 73 new biographies of women mathematicians on Wikipedia.

I also keep an eye on the women included in the "Category: American mathematicians." As of August 30, 2016, there were thirty-seven pages (people) in this category. If we assume every woman in the "Category: American women mathematicians" is also in the "Category: American mathematicians" (which is not the case ${ }^{3}$ ), the percentage of women in the "Category: American mathematicians" was about $17 \%$ at that time. For comparison, according to the AMS Annual Survey, we know that 29\% of the US citizens to earn PhDs from US mathematics departments between 1991 and 2015 were women. As per the 2015 AMS Annual Survey, about 23\% of all full-time doctoral mathematics faculty were women, and 19\% of the Public or Private Large (the top-producing departments) doctoral mathematics faculty were women. I am happy to report that the number of women listed in the "Category: American women mathematicians" nearly doubled between August 2016 and October 2017 (from 37 to 73), in part due to the efforts I'll discuss in the next section.

One deterrent to the creation of more biographies of women mathematicians on Wikipedia is the scarcity of women editors. Another serious impediment is that not all of the articles that are created will "survive" on Wikipedia. Both of these hindrances will be discussed briefly in Section 2. Please see my longer article [3] for more details.

\section{The Scarcity of Women Wikipedia Editors and My Personal Experiences}

Women are substantially underrepresented on Wikipedia as editors (see [2]). A survey conducted by the United Nations University and Maastricht University in collaboration with the Wikimedia Foundation demonstrated that in 2008 only $12.64 \%$ of Wikipedia editors worldwide were women. A follow-up survey in 2011 showed that things hadn't improved: $9 \%$ of editors worldwide and $15 \%$ of those from the US were women. A few years after the initial study, the Wikimedia Foundation announced the goal of raising the proportion of female editors worldwide to 25\% by 2015. In 2014 Wikimedia Foundation founder Jimmy Wales announced that Wikipedia had completely failed to reach this goal despite launching several initiatives. This is particularly disturbing in light of the fact that the percentage of adult Americans who use Wikipedia for information increased from 25\% in February 2007 to $42 \%$ in May 2010, according to a study [1] conducted by the Pew Research Center. The Pew study also showed that about $50 \%$ of the adult female Internet users and $56 \%$ of the male adult Internet users look to Wikipedia for information.

In order to better understand the potential problems an editor faces, I attended my first edit-a-thon on writing women into Wikipedia at the University of Oregon in March of 2013. This event was part of the Wiki Women's History Month events and was led by Sarah Stierch, who

\footnotetext{
${ }^{3}$ It is up to an editor to decide what categories a page belongs to. Sometimes categories that have been assigned by one editor are removed by another editor.
}

was then a program evaluation coordinator for the Wikimedia Foundation and a former Wikipedian in Residence at the Archives of American Art and the Smithsonian Institution Archives.

During the edit-a-thon I decided to write an article about Susan Montgomery. Montgomery was the 2011 AWM Noether Lecturer, and in 2012 she was selected as both an AAAS Fellow and an AMS Fellow in the inaugural class. I felt she clearly merited a page on Wikipedia. I began working on her page during the edit-a-thon and finished up the article a couple of weeks later. My first experience writing for Wikipedia was extremely frustrating, but in the end I was glad that I undertook this project. I learned that there are many Wikipedia conventions that a contributor must follow. I am still learning about these conventions and the culture of Wikipedia.

The first thing I did during the edit-a-thon was to edit the existing Wikipedia page on the Noether Lecture so that the lecturers for 2011-2013, including Montgomery, were listed on that page. I inserted square brackets around Montgomery's name so she appeared as a red link, that is, a person (or topic) mentioned on Wikipedia for whom a Wikipedia page does not exist. ${ }^{4}$ A biography has a better chance of surviving on Wikipedia if the subject is already mentioned on an existing Wikipedia page. I followed all the above-mentioned guidelines and asked Stierch to read my article before I published it. In spite of all my precautions, my article was proposed for deletion with the PROD (Proposed deletion) tag within ten minutes after it went live by a novice editor who made this remark:

It is proposed that this article be deleted because of the following concern: This looks like a case of shameless (self) promotion. Hey, look at me: I teach math and I want my CV on Wikipedia.

Stierch responded to the novice editor by saying the biography was in fact a good faith article by a new editor (not the subject) and removed the PROD tag. The article survived only because of Stierch's intervention. ${ }^{5}$

At Montgomery's request, I started another Wikipedia biography in May of 2014, this one on Georgia Benkart.

\footnotetext{
${ }^{4}$ A Wikipedia editor can easily create a red link on Wikipedia by surrounding [[name]] or [[topic]] with double square brackets as indicated.

${ }^{5}$ Stierch, whose username today is Missvain, is still an active Wikipedia administrator. Regrettably, Stierch lost her Wikimedia Foundation job in late 2013 when she was discovered editing for pay, see arstechnica.com/tech-policy/2014/01/wikimediafoundation-employee-ousted-over-paid-editing, In general there is a prohibition on paid editing, but it isn't uniformly enforced. I have heard that the Ivy League schools have public relations staff who create pages for distinguished faculty; the staff are supposed to acknowledge their employer in their "Talk" pages. I came across one such acknowledgment on the "User" page of Dominic McDevitt-Parks (User: Dominic), who is a paid employee of the US National Archives and Records Administration and edits on Wikipedia for them. I suspect that there are many other paid editors who make no such acknowledgment.
} 
Benkart was AWM President 2009-2011, was elected to the inaugural class of Fellows of the AMS in 2013, and was selected to deliver the AWM-AMS Noether Lecture in 2014 and the Emmy Noether Lecture at the International Congress of Mathematicians in Seoul, Korea, in 2014. She has published over 100 journal articles and has co-authored three Memoirs of the American Mathematical Society. Benkart clearly met the Wikipedia criteria for academic notability. I had Stierch both read my article on Benkart and later monitor it when the article was published on Wikipedia. I also asked Emily Temple-Wood to watch the page and make sure it wasn't deleted.

Soon after the Benkart biography went live, it was tagged for having too many primary sources and not enough secondary sources. Wikipedia recommends that a biography should have at least as many secondary sources in the "References" section as publications of the subject, which are regarded as primary sources. I addressed that criticism and removed the tag after thanking the editor involved for his comments and explaining that most of the sources were now secondary. The article was then tagged for using weasel words: vague phrasing that often accompanies biased or unverifiable information. The Wikipedia administrator asserted the following:

The whole tone of the article is written putting her on a pedestal.

After asking Emily Temple-Wood and Sarah Stierch about weasel words and looking at the "Talk" page for the article, I found out that the administrator was objecting to my claims that Benkart was a "distinguished" mathematician who is an "international leader" and a "renowned" teacher, and for asserting that one of Benkart's joint papers became "one of the building blocks" of the classification of toroidal rank-one Lie algebras. Since I am not an expert in Benkart's research area (the structure and representation theory of Lie algebras), I asked Montgomery for help. Montgomery enlisted the help of Efim Zelmanov, who wrote up the paragraphs that described the "importance" of Benkart's work (I couldn't help slipping in the weasel word "importance"). In this case an administrator who has virtually no background in mathematics objected to a Fields Medalist's description of Benkart's work. As Stierch pointed out to me, this is the reality of the wacky world of Wikipedia. If an editor can't prove her assertion with a reliable secondary source, then any other editor can remove the relevant sentence without question. Stierch removed the uncited weasel words from my article and the tag was eventually removed. As of this writing, the article has survived on Wikipedia.

Other mathematicians have had a difficult time when writing biographies of women mathematicians. For example, Christina Sormani has created several biographies, originally with the username "Sormani." Beginning around 2007 , the articles she wrote were deleted by other editors and Administrators. She then started writing articles anonymously without logging on to the Wikipedia site and had more success. We do not have any data, numerical or anecdotal, on the difficulties editors have in creating or editing pages on male mathematicians.

\section{Steps Toward Improving the Situation}

During the past several years there have been a number of successful attempts to increase the visibility of women mathematicians on Wikipedia. In 2007 the Wikipedia page "Noether Lecture" was created by AWM member Sormani. The Noether Lecture is a lecture series (and award) that honors women "who have made fundamental and sustained contributions to the mathematical sciences." The AWM established the lecture in 1980 and in 2013 it was renamed the AWM-AMS Noether Lecture. The Noether lecturers were all listed on the page Noether Lecture, some as red links (without their own Wikipedia pages). I am happy to report that as of today all of the Noether lecturers listed on the page "Noether Lecture" appear as blue links (with pages on Wikipedia).

The WikiProject Women scientists (Figure 1) began in 2012 and is dedicated to ensuring the quality and quantity of biographies of women in science on Wikipedia. Emily Temple-Wood (who edits on Wikipedia as Keilana) is a co-founder of this project; she started writing Wikipedia pages when she was twelve and continues to write pages and confront gender bias on Wikipedia, although her pace has slowed a bit since she began medical school at Midwestern University in the fall of 2016. Temple-Wood has written over 100 pages on women scientists. She became an administrator in 2007 and is currently part of the Arbitration Committee. There is a plethora of information on the project page for WikiProject Women scientists, including suggestions about how an editor can help the project. There are links to pages that are helpful to Wikipedia editors and a list of more than 80 members of the project. The list of members begins with the usernames of Emily Temple-Wood and Sarah Stierch (Keilana and SarahStierch, respectively); I am member 84 near the bottom of the list.

The objective of the WikiProject Women in Red is to turn red links of women on Wikipedia into blue links.

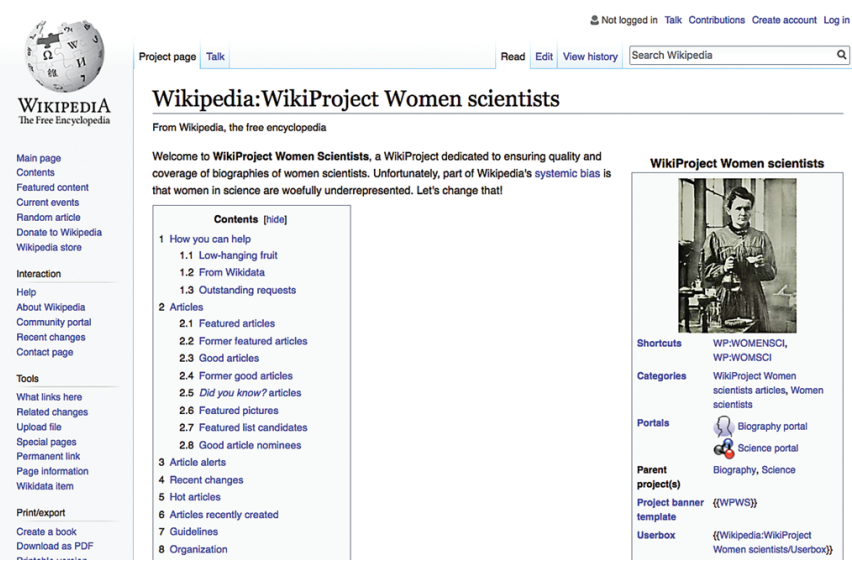

Figure 1. WikiProject Women scientists includes suggestions about how an editor can help. 
Rosie Stephenson-Goodknight (who edits on Wikipedia as Rosiestep) is a co-founder of this WikiProject. WikiProject Women in Red hosts edit-a-thons and publicizes its scope and objective via social media. WikiProject Women in Red has a page on "Mathematics," which is organized by country and lists missing articles on women who are (or have been) notable for their contributions to mathematics in academics, business, economics, politics, research, government, or the social sector. I made many additions to the list and encourage you to do so as well. To locate the pages of these WikiProjects one can use the special search box at en.wikipedia.org/wiki/Wikipedia:WikiProject.

Temple-Wood and Stephenson-Goodknight were named as 2016 co-Wikipedians of the Year by Wikipedia Trustee Jimmy Wales in recognition of their work on harassment on Wikipedia along with their collective efforts to expand coverage of notable women on Wikipedia.

The year 2016 was the Wikipedia Year of Science, an unparalleled initiative to improve scientific literacy through Wikipedia. A fundamental part of the Year of Science was the Women in Science initiative, the stated goal of which was to create or improve coverage of women scientists on Wikipedia. Three 2016 was the Wikipedia Year of Science. groups, WikiProject Women scientists, WikiProject Women in Red, and the Wiki Education Foundation, joined forces to run virtual edit-a-thons during the year to create and improve articles about women scientists in diverse fields, emphasizing a different area each month between February and December. From the parent page "Wikipedia: Year of Science," you can find a list of the featured areas and their home pages, which were designed to encourage Wikipedians to collaborate, share successes, identify articles that need to be written, find subject-specific sources, or initiate any activity that improves coverage of or celebrates women in science. The month of October highlighted computer science, technology, and mathematics, and the corresponding Wikipedia page included a section entitled "Biographies of Mathematicians To Create or Improve," which I authored. In this section, one can find a list of prominent women in mathematics who either don't have Wikipedia pages or whose pages are stubs (pages that need to be expanded to meet Wikipedia standards). The page also contains a section labeled "Association for Women in Mathematics" that lists AWM awards, prizes, and lectures that either don't have pages or some of whose recipients don't have pages. This page is a good starting point for someone who wants to edit or create a page enhancing the visibility of women in mathematics on Wikipedia.

In the recent past, there have been efforts to create pages for women elected as Fellows of the AMS or fellows of other major professional organizations. Formerly, on the "Talk" page of the "WikiProject: Women scientists," in a section entitled "Some missing mathematicians," there was a list of women who had been elected Fellows of the AMS but didn't have pages; soon after the list appeared, pages were created for all of these women, primarily by User:Brirush and User:David Eppstein. This wasn't done for the class of 2018 AMS Fellows. I created a page for Rachel Justine Pries. Almost immediately, another Wikipedia editor, User:FormalDude, attached the PROD tag to the article and proposed it for deletion. I successfully defended the notability of Pries and had a team of editors watch the page; to date the page has survived. Pages for 2018 Fellows Antonella Grassi and Hema Srinivasan were created by Ursula Whitcher and David Eppstein, respectively.

In the summer of 2016 Jami Mathewson of the Wiki Education Foundation created the page "Category: Awards and prizes of the Association for Women in Mathematics." As of October 12, 2017 there were four pages in this category: "AWM/MAA Falconer Lecturer," "Louise Hay Award," "M. Gweneth Humphreys Award," and "Noether Lecture." Some of the mathematicians listed on the first three pages appear as red links. The Wikipedia page Association for Women in Mathematics" lists all past and current AWM presidents. Despite the fact that all AWM presidents have Wikipedia pages, and hence appear as blue links, Mathewson reports that only one page (the page for Ruth Charney) has been rated as "B-class" by the WikiProjects Biography and Women scientists, designating it as one of Wikipedia's higher-quality articles. ${ }^{6}$ Many of the pages for former AWM presidents are stubs. To be fair, many of the pages are not rated. My point in mentioning this assessment is that there are still a lot of opportunities for work for fledgling editors who may not yet want to create a page from scratch.

Several women who have created biographies of women in mathematics on Wikipedia recommend that the author of a new page enlist the aid of existing editors to affirm the new article by making minor edits soon after the article goes live. Edits can be made by less senior editors without logging in to their Wikipedia accounts; only the IP address of the computer they were using will appear on the "History" page of the article. It is even better if a page creator has an active administrator looking out for a newly created article.

While writing this article I learned of an unprecedented partnership between the AWM and the Wiki Education Foundation. There is considerable need for such a partnership, particularly since there don't seem to be many mathematicians involved in the WikiProject Women scientists and the WikiProject Women in Red. As I said earlier, I added my name as a member of the WikiProject Women scientists, but I didn't see any other usernames that I recognized. In the past, I

\footnotetext{
${ }^{6}$ To see these ratings, click on the tabs for the "Talk" pages of the articles.
} 
have sought out Stierch and Temple-Wood for advice and for help protecting the pages I wrote. Neither of these allies has much time to devote to Wikipedia today. In response to a query I posted on Temple-Wood's “Talk" page, Susan Barnum, a public services librarian who lives in El Paso, Texas, and has the username Megalibrarygirl, offered help with copyediting and references. It is crucial that we add women editors who can create pages for prominent women in mathematics. I believe that this effort will fail unless there is a network of Wikipedia editors and administrators who watch the pages that we create. Hopefully the partnership can help build such a network.

In another unprecedented move, AWM member and associate editor at Mathematical Reviews Ursula Whitcher organized a Wikipedia women in mathematics edit-a-thon at the 2017 AWM Research Symposium, which was held on the campus of the University of California, Los Angeles. The edit-a-thon was supported by a grant from the Wiki Education Foundation. Mathewson and I assisted Whitcher during the edit-a-thon. Edit-a-thons are wonderful resources for novice editors. Ten new pages on women mathematicians were created during the AWM edit-a-thon, including a page I created on 2017 AMS Fellow Mei-Chu Chang. Please view and help improve these pages by visiting the Wikipedia page for the edit-a-thon.

\section{Summary}

There are many more Wikipedia articles on women mathematicians today than when I first became a Wikipedia editor in early 2013. However, many of these articles are stubs, and not a single woman mathematician appears in the ten articles allotted to mathematicians in "Wikipedia: Vital_Articles." WikiProject Women scientists is attempting to have the page on Emmy Noether included in this prestigious list. Although all of the women who were elected AMS Fellows now have Wikipedia pages, several of the women who were elected SIAM Fellows or who have received awards from national organizations still do not have pages. The percentage of women editors on Wikipedia remains dismally low.

I have edited many biographies of women mathematicians and will continue to do so. Even though writing a new biography is at times frustrating and is always time consuming, I am glad that I created biographies of four prominent women mathematicians and plan to write more. Anyone who wants to become a Wikipedia editor can find a useful list of Wikipedia help pages on the bottom of the "Project" page for the WikiProject Women scientists. Placing (WPWS) at the top of the "Talk" page of an article will result in the article being automatically added to "Category: WikiProject women scientists" and the other members of the WikiProject will be able to keep an eye on the page. I hope you will decide to take the plunge. Our entire community will be looking forward to reading new articles on notable women in mathematics.
ACKNOWLEDGMENT. I would like to acknowledge and applaud the efforts of Emily Temple-Wood and Rosie Stephenson-Goodknight for their work on harassment on Wikipedia along with their collective efforts to expand coverage of notable women on Wikipedia.

\section{References}

1. KAthryn and LeE RAinie, Wikipedia past and present: A snapshot of Wikipedia users. Pew Research Center. January 13, 2011, www. pewinternet.org/2011/01/13/wikipediapast-and-present.

2. NICOLE TORRES, Why do so few women edit Wikipedia? Harvard Business Review, June 2, 2016, https://hbr.org/2016/06/ why-do-so-few-women-edit-wikipedia.

3. MARIE A. VITULLI, Writing women mathematicians into Wikipedia (long version), 2017, arXiv: 1710.11103v3[math . HO].

Image Credits

Figure 1 from en.wikipedia.org/wiki/Wikipedia: WikiProject_Women_scientists. Photo of Marie A. Vitulli courtesy of Marie A. Vitulli.

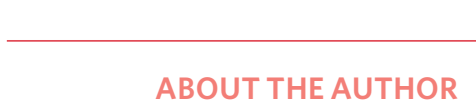

Marie A. Vitulli's main research interests are in commutative algebra and algebraic geometry, particularly in the theories of seminormality, weak normality, and valuations on commutative rings. She has been concerned about the underrepresentation of women in mathematics and the inadequate recognition of outstanding female mathematicians since the late 1970s when she began her long career as a university professor of mathematics. 